\title{
A Word from USDA APHIS in response to the March Protocol Review
}

To the Editor - In response to the March Protocol Review column, "Showtime! Should an IACUC oversee companion animal exhibition activities on campus?" the United States Department of Agriculture Animal and Plant Health Inspection Service (USDA APHIS) provides the following clarification:

In this scenario, a university-supported club overseen by an experienced faculty advisor provides informal instruction on handling, grooming, and training of pet dogs and cats for the purpose of participation in dog and cat shows and dog agility competitions. Although the term "animal” includes dogs used for research, testing, experimentation, or exhibition purposes, or as a pet, the Animal Welfare Act (AWA) regulations specifically exclude purebred dog and cat shows, field trials, and coursing events from coverage $e^{1,2}$.

In addition, the USDA does not consider activities such as grooming and training associated with purebred dog and cat shows, field trials, and coursing events to constitute research, testing, or experimentation as those terms are used in the definition of "research facility." Because the dogs are not used in research or intended for use in research, the Institutional Animal Care and Use Committee is not required to oversee the activities.

\section{Bernadette Juarez}

Deputy Administrator, Animal Care, APHIS, USDA, Riverdale, MD, USA.

e-mail:bernadette.r.juarez@aphis.usda.gov

Published online: 11 March 2019

https://doi.org/10.1038/s41684-019-0267-0

References
1. 7 U.S.C. $\$ 2132$
2. 9 C.F.R. $\$ 1.1$ 